\title{
Unmasking molecular mechanisms
}

DOI:

10.1038/nrd2298
Despite advances in the therapy of heart failure - such as beta blockers and renin-angiotensin antagonists — there is still a need for better therapies for many patients with this condition. Two recent papers have uncovered some of the molecular mechanisms involved in this disease, which could aid in the search for new therapeutic targets.

In the first study, published in PLoS Biology, Wang and colleagues investigated the mechanisms underlying cardiac calcium-induced calcium release (CICR), a process in which calcium influx through cell-surface L-type calcium channels (LCC) activates ryanodine receptor (RyR) calcium release from the sarcoplasmic reticulum to generate cell-wide calcium transients. This process governs the contractile power of the heart, and might undergo progressive dysregulation in cardiac hypertrophy.

The authors used patch clamping and confocal calcium imaging to visualize CICR in cardiac myocytes from rat models of compensated hypertrophy (a precursory stage of heart failure in which the heart produces an adaptive response) and decompensated hypertrophy (an early stage of heart failure in which the heart undergoes functional deterioration). The authors found that the intermolecular-coupling efficiency between LCC and RyR was compromised in decompensated hypertrophy; there was a prolongation in coupling latency, accompanied by a decrease in the chance of CICR occurring. At a whole-cell level calcium release from RyR became desynchronized. Surprisingly, the intermolecular-coupling efficiency between LCC and RyR was also compromised in compensated hypertrophy, even though at a whole-cell level, cell-wide calcium transients remained unaffected. The molecular changes in compensated hypertrophy were in part due to decreased expression of junctophilin - a protein that anchors the sarcoplasmic reticulum to the cell membrane.

These results suggest that in heart failure, there is a state of intermolecular failure that proceeds without an alteration in cellular function. So, targeting this period at a very early stage of the condition - perhaps by LCC inactivation or regulation of junctophilin - suggests that it might be beneficial in the prevention of heart failure.

In the second study, Koch and colleagues, writing in Nature Medicine, investigated the regulation of the adrenal $\alpha_{2}$ adrenergic receptors $\left(\alpha_{2}\right.$ ARs) in heart failure, a condition in which there is increased sympathetic nervous system activity, reflected by elevated catecholamine levels.

In adrenal medulla chromaffin cells from rat and mouse models of heart failure, the authors observed lower $\alpha_{2}$ AR density and increased levels of G-protein-coupled receptor kinase 2 (GRK2) - a kinase involved in receptor desensitization. In these cells, an $\alpha_{2}$ AR-specific agonist was unable to attenuate nicotine-induced adrenaline and noradrenaline secretion, showing that in addition to $\alpha$-receptor downregulation, the remaining receptors were functionally uncoupled. Delivery

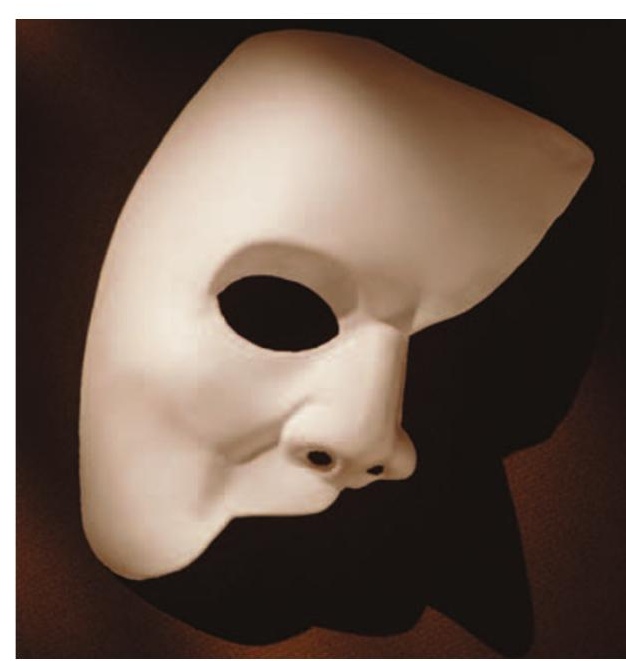

of an inhibitor of GRK2 activation $\beta$-adrenergic-receptor-kinase inhibitor $(\beta$ ARKct $)$ - led to restoration of agonist-mediated inhibition of adrenaline and noradrenaline secretion. When $\beta A R K c t$ was injected into the adrenal glands of rats following myocardial infarction, there was a decrease in adrenal GRK2 levels and a reduction in circulating levels of adrenaline and noradrenaline, accompanied by an enhanced cardiac function. Overall, these results indicate that adrenal GRK2 inhibition leads to a reduction of sympathetic stimulation in heart failure, which in turn improves cardiac adrenergic responsiveness. Therefore, GRK2 inhibition could represent a potential sympatholytic strategy for heart-failure treatment.

Charlotte Harrison

ORIGINAL RESEARCH PAPERS $X u$, M. et al. Intermolecular failure of $\mathrm{L}$-type $\mathrm{Ca}^{2+}$ channel and ryanodine receptor signalling in hypertrophy. PLoS Biol. 5, e21 (2007) | Lymperopoulos, A. et al. Adrenal GRK2 upregulation mediates sympathetic overdrive in heart failure. Nature Med. 13, 315-323 (2007)

FURTHER READING Kaye, D.M. et al. Drug discovery for heart failure: a new era or the end of the pipeline? Nature Rev. Drug Discov. 6 , 127-139 (2007) 